\title{
Observing the Observer: experiences with contextual inquiry to design a horse rider assessment interface
}

\author{
Elizabeth A. Gandy \\ University of Sunderland \\ Faculty of Computer Science \\ St Peter's Way \\ Sunderland SR6 0DD \\ liz.gandy@sunderland.ac.uk
}

\author{
Sharon McDonald \\ University of Sunderland \\ Faculty of Computer Science \\ St Peter's Way \\ Sunderland SR6 ODD \\ sharon.mcdonald@sunderland.ac.uk
}

\author{
Robert Hogg \\ University of Sunderland \\ Faculty of Health Sciences and Wellbeing \\ City Campus, Chester Road \\ Sunderland SR1 3SD \\ bob.hogg@sunderland.ac.uk
}

\author{
Timothy M.C. Pigott \\ University of Salford \\ School of Health Sciences \\ Allerton Building, Salford \\ Greater Manchester M6 6PU \\ t.pigott@salford.ac.uk
}

\begin{abstract}
Current approaches to the postural assessment of horse riders is highly subjective, with a lack of consistency between practitioners. A technology-based solution would remove the human perspective and enable a more consistent, accurate and objective assessment to be carried out. This paper provides preliminary insights on the use of a customised method based on contextual inquiry for gathering contextual data in an unusual, in the wild context. This will be used to determine a standardised data collection protocol and context-specific interface design for an inertial motion sensor based rider assessment tool.
\end{abstract}

Contextual inquiry. Context of use. Data visualisation. Research in the wild.

\section{INTRODUCTION}

$\mathrm{HCl}$ research has been an advocate for gathering data in the wild for many years now, in order to investigate user needs (Chamberlain et al., 2012). In this paper, we present an approach to gathering data in a very unusual context that really is in the wild; the postural assessment of horse riders.

Current techniques for postural assessment of horse riders are based on subjective observation by equestrian practitioners, video analysis or the emerging use of commercial products with a lack of scientific evidence to support their effectiveness. This paper provides preliminary insights into the contextual analysis phase of a project which aims to design a software tool that will use automated inertial motion sensor (IMU) technology to generate visualisations of rider posture. This tool can be used by coaches to present rider assessment feedback and to provide evidence of postural issues for referral to other practitioners, such as physiotherapists, who may not be available to carry out a face-to-face assessment at the time of riding.

We suggest a protocol for a customised version of contextual inquiry, appropriate for carrying out contextual analysis specifically in situations where the participant is themselves engaged in observational activity. We will discuss the use of this method to identify the tasks to be incorporated into a standardised rider assessment protocol, the biomechanical factors to be obtained from the IMU data and appropriate visualisations to present meaningful results within the context of use.

\section{BACKGROUND}

The incidence rate of low back pain in equestrian sports is reported as 3-5 times greater than that of the general population, with asymmetrical posture identified as a primary cause (Nevison \& Timmis, 2013). There is a need to find a method of measuring this accurately and consistently (Gandy et al., 2014). Preliminary investigation has indicated that IMU technology may provide a practical solution but current analysis software is complex and further research is required to determine appropriate data visualisations for this context of use (Gandy et al., 2014). Previous studies have focused on subsets of biomechanical measures, movements and gaits whereas a more holistic approach is required for assessment by practitioners (Blokhuis et al., 2008).

Despite the popularity of commercial sensor-based assessment tools across health, sport and educational contexts, there is a lack of scientific evidence to support software design choices and a need for further research into feedback mechanisms used (Schneider et al., 2015).

The assessment of the context in which the tool sits is important because we have different agents 
(rider, coach and physiotherapist) with different needs and motivations. The tool must fit the context, work in different locations and meet the expectations of these different users.

There is a need, therefore, to examine contextual factors such as the tasks riders are required to perform during the assessment, the thinking that informs the strategies employed by the coach whilst observing rider posture and the information required for referral on to physiotherapists or other practitioners.

\section{METHOD}

Due to the observational nature of rider assessment, the software requirements elicitation process needs to be carried out in the wild, without interfering with the user. The method selected must address the challenge of carrying out an observation of a user (the coach) who is themselves carrying out an observation of a third party (the rider).

Field study techniques enable deep insight to be gained into the tasks and processes carried out by users within their normal daily work or living (Rosenbaum, \& Kantner, 2007). In our context, only the assessment aspect of the coach's role is being investigated so a customised version of the Condensed Contextual Inquiry method proposed by Rosenbaum and Kantner (2007) has been selected. Observation and interpretation phases, using think-aloud recall to enable the thought processes of the coach to be recorded, will determine the assessment protocol and biomechanical measures to be incorporated into the proposed tool. A structured interview is then used to identify appropriate interface designs for visualisation of the IMU data.

\subsection{Participants}

Following a pilot session to confirm the viability of the method, preliminary data collection was carried out with two coaches, both qualified to BHSAI (British Horse Society Assistant Instructor) standard. The same adult rider was assessed by each coach separately but on different horses. The rider and horses were selected as capable of performing at British Dressage novice standard.

All ethical and health and safety consents were obtained prior to data collection.

\subsection{Observation of coach assessment}

The coach was directed to carry out a visual postural assessment of a rider, as would typically be carried out at the start of a coaching session, with a guideline time of 10-20 minutes. The coach was allowed to move freely within the arena and to interact with the rider to provide instructions on the tasks selected to be observed but they were asked to provide minimal coaching cues on rider posture.

The assessment was recorded using a GoPro HERO4+ ${ }^{\text {TM }}$ head-mounted camera to capture the coach's viewpoint and a Sony digital video camcorder positioned at one end of the arena to capture both coach and rider's location and movements (Figure 1).

The researcher observed the assessment in silence to avoid interruption to the coach.

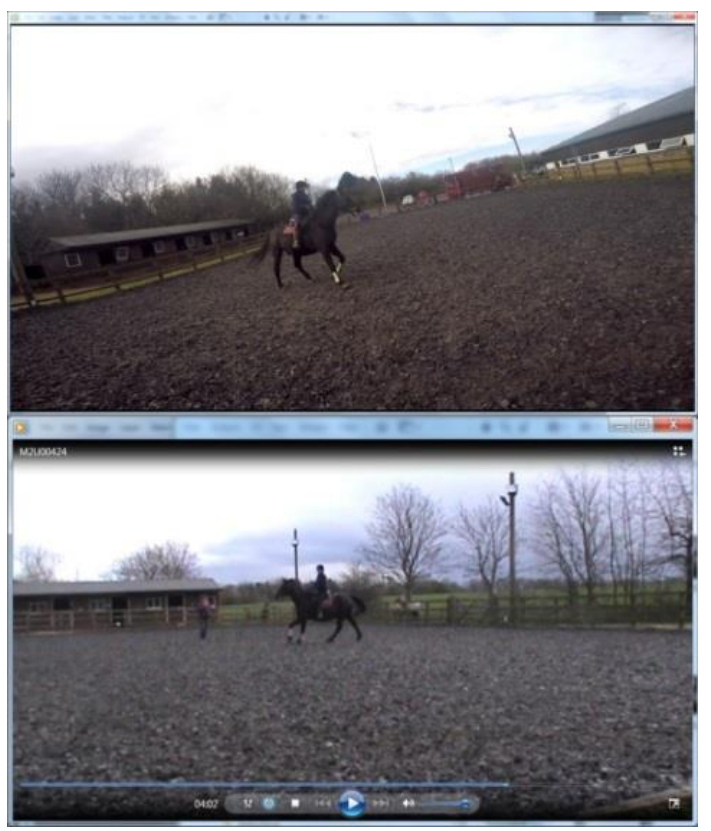

Figure 1. Coach (top) and researcher (bottom) views captured during the rider assessment.

\subsection{Retrospective Think-aloud (RTA)}

A RTA was used to enable the coach to provide explanations of their thought processes during the observation phase, to determine the biomechanical markers and measures to be incorporated into the proposed tool.

The video files from the assessment phase were transferred to a laptop computer and Windows Media Player ${ }^{\mathrm{TM}}$ used to play back the GoPro ${ }^{\mathrm{TM}}$ recording to assist the coach during the recall.

The coach was given control of the laptop so that they could pause and/or repeat sections of the video as required while they described their thought processes during the rider assessment. The laptop screen and microphone were recorded using Camstudio TM so that the GoPro TM playback could be aligned with the retrospective recall for subsequent analysis. The coach again wore the head-mounted GoPro TM camera to provide a backup recording.

The coach was instructed to explain the reasons for the specific tasks they had asked the rider to 
perform and the areas of the body that they were focusing on during each task.

The researcher did not interrupt the coach during the recall phase beyond reminding then to thinkaloud. Questions identified were asked at the end of the recall phase, before stopping the screen/audio recording.

\subsection{Structured Interview}

A recorded structured interview followed, during which the researcher asked additional questions relating to the proposed software tool. The coach was shown a variety of interface design possibilities and hand-drawn sketches were made by the researcher to support the discussion (Figure 2).

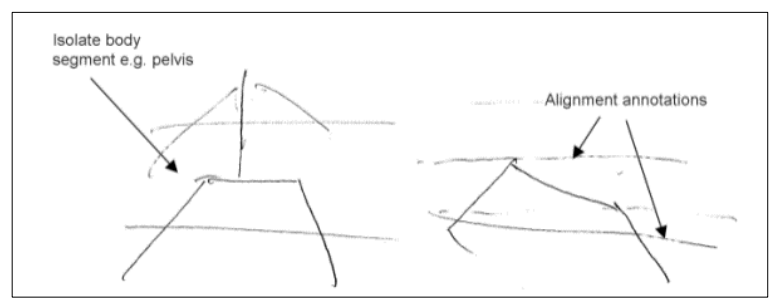

Figure 2. Sketches drawn by the researcher to support discussions during the structured interview (Coach 1).

The structured interview was concluded with a request for feedback and suggestions for future improvement of the data collection method used.

\subsection{Data Analysis}

The video recordings of the observation phase were coded according to standardised riding movement descriptors and gaits of the horse. Future work will investigate patterns and commonality between coaches to determine a standardised protocol for rider assessment. The RTA phase was used to code the biomechanical markers considered important for each task according to the Xsens TM IMU technology data format for body segments and joint angles. This was cross-checked by a biomechanics expert to ensure variations in phrases used were correctly interpreted.

Qualitative analysis was carried out on the structured interview responses to determine interface design and data visualisation preferences.

\section{PRELIMINARY RESULTS AND DISCUSSION}

Analysis is currently ongoing so we will focus our discussion on the structured interview responses relating to interface design and data visualisation.

\subsection{Anatomical Visualisation}

The coaches were initially shown anatomical representations of the rider from the proprietary software distributed with the Xsens TM system (Figure 3). Both responded positively, with Coach 1 commenting "If the flesh was taken off and you've just got the skeletal system it makes it much easier. Yes, definitely. I'd love something like that, I really would".

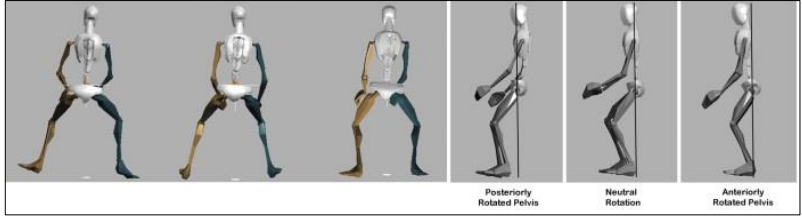

Figure 3. Screen captures from XSens MVN Studio ${ }^{T M}$, with added annotations

The rear view was considered useful for assessing asymmetry of the pelvis, shoulders and head. Coach 1 suggested functionality to isolate body segments such as the pelvis and the addition of annotations to show optimal alignment (Figure 2). Coach 2, preferred simplicity and felt that only the vertical shoulder/hip/heel line was necessary, as in the sagittal view images in Figure 3, with no need for a horizontal line showing pelvic alignment.

Figure 4 shows an alternative anatomical visualisation provided to the coaches as an example of how exported IMU data might be incorporated into the proposed tool.

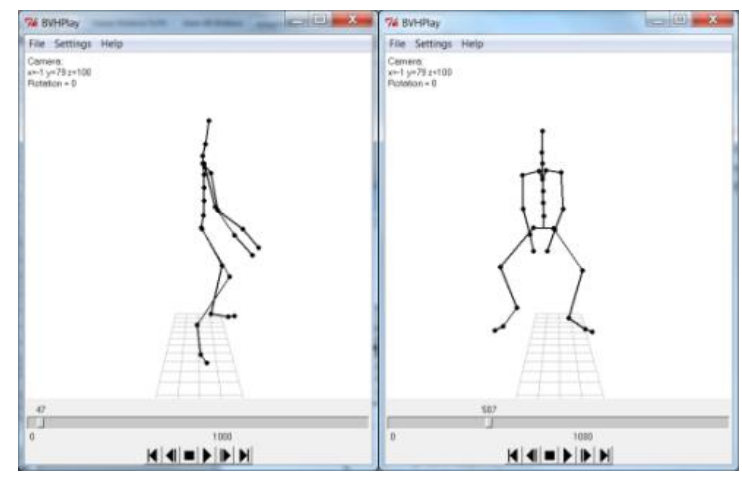

Figure 4. Screen captures from BVHPlay ${ }^{T M}$ showing rear and sagittal views of IMU data in BVH format.

Both coaches found the rear view clear but the sagittal view confusing: it was difficult to distinguish between left and right limbs. Coach 1 suggested either separate views or use of colour to distinguish each side. Coach 2 preferred 4 separate views (left, right, front, rear), displayed simultaneously, rather than colour. She had no preference on layout, either a line of 4 images or a 2x2 grid.

The use of "traffic light" colour coding was suggested to show divergence from correct posture or intra-rider progression between sessions. Coach 1 felt that agreement on colour boundaries would be difficult, requiring consultation with a large number of coaches to determine optimal boundaries. Coach 2 felt there was little benefit to automation of postural fault detection, preferring 
personal interpretation and thought colour labelling in red might have a negative impact on sensitive riders. Following a discussion of animation versus still snapshots, she concluded that a coloured traffic light system on a fast moving animation could reduce the chance of missing critical detail.

\subsection{Graphical Representation}

A graphical display of rider hip rotation asymmetry was shown (Figure 5) to gauge opinion on the use of charts to visualise data analysis.

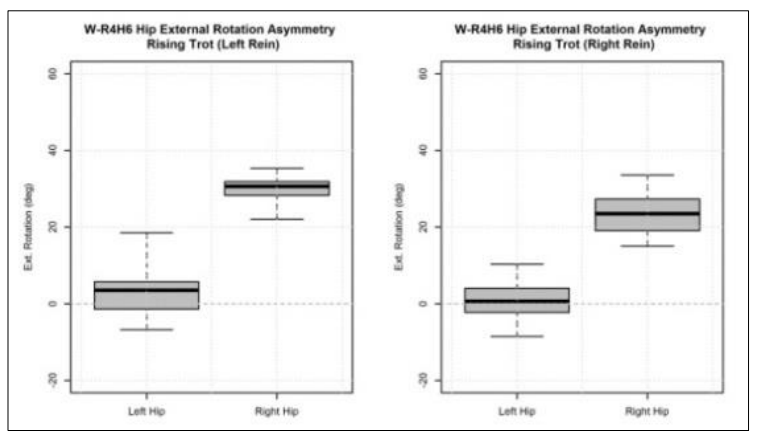

Figure 5. Example of a graphical representation of rider hip rotation asymmetry.

Both coaches found this to be confusing and required significant explanation, preferring the simplicity of the "stick figure" previously discussed.

\subsection{Numerical data representation}

A screen capture from a commercial bike fit system (Retul TM) was used as an example of a design incorporating numerical data (Figure 6).

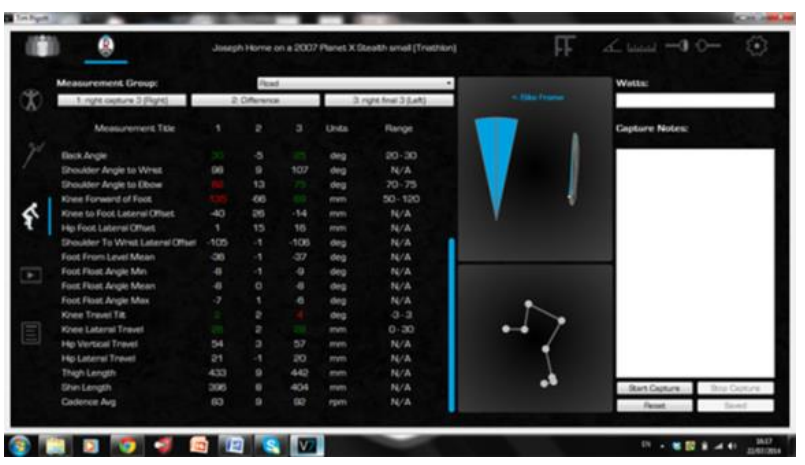

Figure 6. Screen capture from Retul ${ }^{T M}$ bike fit system

Both coaches found this less intuitive than the anatomical visualisations. Coach 1 thought it could be useful for competition riders, although Coach 2 disagreed. Both coaches thought numerical data could be of benefit for disabled riders, particularly when liaising with physiotherapists. Coach 2 suggested it could be useful to show changes such as reduced muscle tightness between the start and end of a riding session and as evidence of the benefits of riding therapy across multiple sessions.

\subsection{Reporting}

Both coaches thought that a summary take home report would be useful. Coach 2 commented "people don't always take everything in, so something to take home and have a look at that they could assess it themselves in their own time".

Reports were also considered useful for referrals to other practitioners. Coach 1 previously used photographs for this purpose and noted that the proposed tool would provide more detail.

Both emphasised the importance of customisation, as the focus would be on different aspects for each rider, but Coach 2 suggested a very basic layout with still images showing general posture, shoulder/hip/heel line annotation and an indication of any asymmetries.

\section{CONCLUSIONS AND FURTHER WORK}

While still at a preliminary stage, with further data collection planned, we have found condensed contextual inquiry to be an effective method for use in this context. Both coaches reported that it was easier to carry out the rider assessment without interruption during the observation phase and that the playback of the GoPro ${ }^{\mathrm{TM}}$ recording helped their thought processes during the RTA. We are already seeing patterns that will help us to determine the protocol and biomechanical measures for the proposed IMU-based rider assessment tool.

The example designs gave the coaches a flavour of data visualisations that might be possible and helped to stimulate discussion. It is clear that anatomical visualisation is a likely preferred interface design but further work will be the development and evaluation of prototype software designs incorporating annotations, colour, numerical representation and report formats.

\section{REFERENCES}

Blokhuis, M. Z., Aronsson, A., Hartmann, E., Van Reenen, C. G., \& Keeling, L. (2008). Assessing the rider's seat and horse's behavior: difficulties and perspectives. J Appl Anim Welf Sci, 11(3), 191-203.

Chamberlain, A., Crabtree, A., Rodden, T., Jones, M., \& Rogers, Y. (2012). Research in the wild: understanding 'in the wild' approaches to design and development. Designing Interactive Systems Conference, Newcastle Upon Tyne, United Kingdom. June 2012, 795-796. ACM.

Gandy, E. A., Bondi, A., Hogg, R., \& Pigott, T. M. C. (2014) A preliminary investigation of the use of inertial sensing technology for the 
measurement of hip rotation asymmetry in horse riders. Sports Technology, 7(1-2), 79-88.

Nevison, C. M., \& Timmis, M. A. (2013) The effect of physiotherapy intervention to the pelvic region of experienced riders on seated postural stability and the symmetry of pressure distribution to the saddle: A preliminary study. Journal of Veterinary Behavior: Clinical Applications and Research, 8(4), 261-264.

Rosenbaum, S., \& Kantner, L. (2007) Field Usability Testing: Method, Not Compromise. IPCC 2007. Seattle, USA, October 2007, 1-7. IEEE International.

Schneider, J., Borner, D., van Rosmalen, P., \& Specht, M. (2015) Augmenting the senses: a review on sensor-based learning support. Sensors (Basel), 15(2), 4097-4133. 